Tomasz Natkaniec, Mathematical Institute WSP, Chodkiewicza 30, 85-064

Bydgoszcz, Poland, e-mail: wspb11@@pltumk11.bitnet

\title{
EXTENDABILITY AND ALMOST CONTINUITY
}

\section{Sometimes it's as easy to prove a stronger result ...}

Kenneth R. Kellum

\begin{abstract}
Every function $f:[0,1] \rightarrow[-1,1]$ can be expressed as the sum of three extendable functions, as the maximum of two minimums of extendable functions and as the limit of a transfinite sequence of extendable functions.
\end{abstract}

Let us establish some terminology to be used. Let $I=[0,1]$ and let $X, Y$ be topological spaces. A function $f: X \rightarrow Y$ is:

- Darboux if it maps connected sets onto connected sets,

- almost continuous if every open neighborhood of $f$ in $X \times Y$ contains a continuous function from $X$ into $Y$,

- connectivity if the restriction $f \mid C: C \rightarrow Y$ is a connected subset of $X \times Y$ whenever $C$ is a connected subset of $X$,

- extendable if there is a connectivity function $F: X \times I \rightarrow Y$ such that $F(x, 0)=f(x)$ when $x \in X$.

For $X=Y=I$ we have the following chain of proper inclusions ([15], [12]): continuous $\subset$ extendable $\subset$ almost continuous $\subset$ connectivity $\subset$ Darboux

It is known that a function $F: I \times I \rightarrow \mathbb{R}$ is connectivity iff it is

Key Words: extendable function, connectivity function, almost continuous function, peripherally continuous function, $g$-negligible set

Mathematical Reviews subject classification: Primary: 26A15; Secondary: 54C08

Received by the editors March 7, 1995 
- peripherally continuous, i.e., if for every $x \in I^{2}$ and all open neighborhoods $U$ of $x$ and $V$ of $F(x)$, there exists an open neighborhood $W$ of $x$ in $U$ such that $F($ bd $W) \subset V[6]$.

Let $\mathcal{K}$ denote a class of functions from $I$ into $I$ and let $g \in \mathcal{K}$. A set $M \subset I$ is called $g$-negligible with respect to $\mathcal{K}$ if $f \in \mathcal{K}$ whenever $f: I \rightarrow I$ and $f=g$ on $I \backslash M$ ([1], see also [13]). This is the same as saying that every function $f: I \rightarrow I$ obtained by arbitrarily redefining $g$ on $M$ is still a member of $\mathcal{K}$.

Many theorems on representations of real functions as sums, products, maximums and minimums, or limits of sequences of almost continuous (and therefore also of connectivity and Darboux) functions can be proved using the Kellum \& Garret's method of intersecting of blocking sets ([9], see also [11]). The analogous method does not work in the case of extendable functions. However, the latest results of Rosen on negligible sets with respect to the class of extendable functions can be applied to obtain some related results. Rosen showed how to express an arbitrary real function $f: I \rightarrow I$ as the pointwise limit of a sequence of extendable functions and an arbitrary $g: I \rightarrow$ $[-1,1]$ as the sum of an infinite series of extendable functions [13]. We shall prove that every function $f:[0,1] \rightarrow[-1,1]$ can be expressed as the sum of three extendable functions, as the maximum of two minimums of extendable functions and as the limit of a transfinite sequence of extendable functions.

We need several lemmas. The first of them is obvious. (See e.g. [7].)

Lemma 1 Assume that $X, Y$ and $Z$ are topological spaces, $h: X \rightarrow Y$ is a homeomorphism and $f: Y \rightarrow Z$ is connectivity. Then $f \circ h$ is a connectivity function.

Corollary 1 If a function $f: I \rightarrow I$ is extendable and $h: I \rightarrow I$ is a homeomorphism, then $f \circ h$ is extendable.

Lemma 2 Assume that $J$ is a compact interval, $g: I \rightarrow J$ is an extendable function, $h: I \rightarrow I$ is a homeomorphism, and $A$ is a g-negligible set with respect to the class of extendable functions. Then $h^{-1}(A)$ is $(g \circ h)$-negligible.

Proof. Assume that $g_{1}=g \circ h$ and $\left[f_{1} \neq g_{1}\right] \subset h^{-1}(A)$ for some $f_{1}: I \rightarrow J$. Put $f=f_{1} \circ h^{-1}$. Then $[f \neq g] \subset A$. Indeed, if $x \notin A$, then $h^{-1}(x) \notin h^{-1}(A)$. Therefore $f_{1}\left(h^{-1}(x)\right)=g_{1}\left(h^{-1}(x)\right)$, so $f(x)=g(x)$. Thus $f$ is extendable to a connectivity function and $f_{1}=f \circ h$ is extendable, too.

Lemma 3 Assume that $A, B \subset(0,1)$ are of the first category. Then there exists a homeomorphism $h: I \rightarrow I$ such that $B \cap h(A)=\emptyset$. 
Proof. We may assume that $A$ and $B$ are $F_{\sigma}$ sets and $A=\bigcup_{n=1}^{\infty} F_{n}$, where $F_{n}$ is closed and $F_{n} \subset F_{n+1}$ for $n \in \mathbb{N}$. Moreover, we may assume that each $x \in F_{n}$ is a point of bilateral accumulation of $F_{n+1}$, and $\left|J_{0}\right| \leq|J| / 2$ whenever $J$ is a component of the set $I \backslash F_{n}$ and $J_{0}$ is a component of $J \backslash F_{n+1}(|J|$ denotes the length of an interval $J$ ). For every $k \in \mathbb{N}$ let $\left(I_{k, n}\right)_{n}$ be a sequence of all components of the set $I \backslash F_{k}$.

Since the set $I \backslash(B \cup\{0,1\})$ is a dense $G_{\delta}$ subset of $I$, there exists a Cantor set $E_{1} \subset I \backslash(B \cup\{0,1\})$ such that $|J| \leq 1 / 2$ for each component $J$ of the complement of $I \backslash E_{1}$. Let $h_{1}: I \rightarrow I$ be an increasing homeomorphism such that

- $h_{1}\left(F_{1}\right)=E_{1}$ (hence $h_{1}\left(F_{1}\right) \cap B=\emptyset$ ),

- $h_{1}$ is linear on each $\bar{I}_{1, n}$,

- $\left|h_{1}\left(I_{1, n}\right)\right| \leq 1 / 2$ for every $n \in \mathbb{N}$.

For each $n \in \mathbb{N}$ let $J_{1, n}=h_{1}\left(I_{1, n}\right)$ and let $h_{2, n}: \bar{I}_{1, n} \rightarrow \bar{J}_{1, n}$ be an increasing homeomorphism such that

- $h_{2, n}\left(I_{1, n} \cap F_{2}\right) \cap B=\emptyset$,

- $h_{2, n}\left(\right.$ bd $\left.I_{1, n}\right)=h_{1, n}\left(\operatorname{bd} I_{1, n}\right)$,

- $h_{2, n}$ is linear on the closure of every component of $I_{1, n} \backslash F_{2}$,

- $\left|h_{2, n}(J)\right| \leq\left|J_{1, n}\right| / 2$ for every component $J$ of $I_{1, n} \backslash F_{2}$.

Note that $h_{2}=\bigcup_{n} h_{2, n}$ is an increasing homeomorphism of $I$ onto $I$ and $E_{2}=h_{2}\left(F_{2}\right)$ is disjoint from $B$. Assume that for $k \in \mathbb{N}$ we have defined an increasing homeomorphism $h_{k}: I \rightarrow I$ such that $E_{k}=h_{k}\left(F_{k}\right)$ is disjoint from $B$. For each $n \in \mathbb{N}$ let $J_{k, n}=h_{k}\left(I_{k, n}\right)$ and let $h_{k+1, n}: \bar{I}_{k, n} \rightarrow \bar{J}_{k, n}$ be an increasing homeomorphism such that

- $h_{k+1, n}\left(I_{k, n} \cap F_{k+1}\right) \cap B=\emptyset$,

- $h_{k+1, n}\left(\operatorname{bd} I_{k, n}\right)=h_{k, n}\left(\operatorname{bd} I_{k, n}\right)$,

- $h_{k+1, n}$ is linear on the closure of every component of $I_{k, n} \backslash F_{k+1}$,

- $\left|h_{k+1, n}(J)\right| \leq\left|J_{k, n}\right| / 2$ for every component $J$ of $I_{k, n} \backslash F_{k+1} \cdot \mathrm{n}$

Let $h_{k+1}=\bigcup_{n} h_{k+1, n}$ and $E_{k+1}=h_{k+1}\left(F_{k+1}\right)$. Observe that the function $\widetilde{h}=\bigcup_{k}\left(h_{k} \mid F_{k}\right)$ is increasing, the set $A$ is dense in $I$, and for each $x \in I$ we can define $h(x)=\lim _{t \rightarrow x} \widetilde{h}(t)$. Then $h$ is an increasing homeomorphism from $I$ onto $I$. Moreover, $h(A) \cap B=\emptyset$. 
Theorem 1 For every function $f: I \rightarrow[-1,1]$ there exist three extendable functions $f_{i}: I \rightarrow[-1,1], i=0,1,2$, such that $f=f_{0}+f_{1}+f_{2} \cdot{ }^{1}$

Proof. Let $F: I^{2} \rightarrow I$ be a connectivity function such that for some $x_{0} \in$ $I$ the restriction $d=F \mid\left(I \times\left\{x_{0}\right\}\right)$ is a connectivity function whose graph is dense in $I \times\left\{x_{0}\right\} \times I$; see [12, Example 2]. (Such functions have been constructed in fact in [2] and [4].) Obviously $d$ is an extendable function, so there exists a dense extendable function $g: I \rightarrow[-1,1]$ (cf, [13]). There exists a first category set $A \subset I$ such that $I \backslash A$ is $g$-negligible [13, Theorem 1(iii)]. Since every nowhere dense subset of $I$ is $g$-negligible [13, Theorem 1(i)], we can assume that $A \subset(0,1)$. By Lemma 3, there exist: a homeomorphism $h_{1}: I \rightarrow I$ such that $h_{1}(A) \cap A=\emptyset$, and a homeomorphism $h_{2}: I \rightarrow I$ such that $h_{2}(A) \cap\left(A \cup h_{1}(A)\right)=\emptyset$. By Corollary 1, the functions $g_{i}=g \circ h_{i}^{-1}$, $i=1,2$, are extendable. Define $f_{i}: I \rightarrow[-1,1]$ for $i=0,1,2$ by

$$
\begin{aligned}
& f_{0}(x)=\left\{\begin{aligned}
g(x) & \text { for } x \in A, \\
-g_{1}(x) & \text { for } x \in h_{1}(A), \\
f(x) & \text { for } x \notin A \cup h_{1}(A),
\end{aligned}\right. \\
& f_{1}(x)=\left\{\begin{aligned}
-g(x) & \text { for } x \in A, \\
g_{1}(x) & \text { for } x \in h_{1}(A), \\
-g_{2}(x) & \text { for } x \in h_{2}(A), \\
0 & \text { for } x \notin A \cup h_{1}(A) \cup h_{2}(A),
\end{aligned}\right. \\
& f_{2}(x)=\left\{\begin{aligned}
f(x) & \text { for } x \in A \cup h_{1}(A), \\
g_{2}(x) & \text { for } x \in h_{2}(A), \\
0 & \text { for } x \notin A \cup h_{1}(A) \cup h_{2}(A) .
\end{aligned}\right.
\end{aligned}
$$

By Lemma 2, the functions $f_{i}, i=0,1,2$, are extendable. Moreover, $f=f_{0}+f_{1}+f_{2}$.

Theorem 2 For every function $f: I \rightarrow I$ there exist four extendable functions $f_{i}, i=0,1,2,3$, such that $f=\max \left(\min \left(f_{0}, f_{1}\right), \min \left(f_{2}, f_{3}\right)\right) .^{2}$

Proof. Let $g: I \rightarrow I$ be a dense extendable function and let $A$ be a first category set such that $I \backslash A$ is $g$-negligible. Let $h_{1}: I \rightarrow I$ be a homeomorphism such that $h_{1}(A) \cap A=\emptyset$. Let $h_{2}: I \rightarrow I$ be a homeomorphism such that $h_{2}(A) \cap\left(A \cup h_{1}(A)\right)=\emptyset$. Let $h_{3}: I \rightarrow I$ be a homeomorphism such that

\footnotetext{
${ }^{1}$ The analogous result for almost continuous functions is proved in [3].

${ }^{2}$ The analogous result on almost continuous functions is proved in [10].
} 
$h_{3}(A) \cap\left(A \cup h_{1}(A) \cup h_{2}(A)\right)=\emptyset$. Moreover, let $h_{0}=$ id ${ }_{I}$ and let $g_{i}=g \circ h_{i}^{-1}$ for $i=0,1,2,3$. By Corollary 1 , the functions $g_{i}$ are extendable. Define

$$
f_{i}(x)=\left\{\begin{array}{rr}
g_{i}(x) & \text { for } x \in h_{i}(A) \\
f(x) & \text { for } x \notin h_{i}(A)
\end{array}\right.
$$

By Lemma 2, all functions $f_{i}$ are extendable. It is easy to verify that $f=$ $\max \left(\min \left(f_{0}, f_{1}\right), \min \left(f_{2}, f_{3}\right)\right)$.

Recall that a function $f: I \rightarrow I$ is a discrete limit of a net $\left(f_{\sigma}\right)_{\sigma \in \Sigma}$, where $(\Sigma, \preceq)$ is a directed set, if for each $x \in I$ there exists $\sigma_{0} \in \Sigma$ such that $f_{\sigma}(x)=f(x)$ whenever $\sigma_{0} \prec \sigma$. Moreover, if $\Sigma=\omega_{1}$, then $f$ is called the limit of a transfinite sequence $\left(f_{\alpha}\right)_{\alpha<\omega_{1}}$ [14].

Let add $(\mathcal{K})$ denote the additivity of the ideal of all first category sets in $I$, i.e., the least cardinal $\kappa$ for which there exists a family $\mathcal{F}$ of first category sets such that $\operatorname{card}(\mathcal{F})=\kappa$ and the set $\bigcup \mathcal{F}$ is of the second category.

Theorem 3 Assume that $(\Sigma, \preceq)$ is a directed set with $\operatorname{card}(\Sigma) \leq \operatorname{add}(\mathcal{K})$. Then each function $f: I \rightarrow I$ is the discrete limit of a net $\left(f_{\sigma}\right)_{\sigma \in \Sigma}$ of extendable functions. ${ }^{3}$

Proof. Let $g: I \rightarrow I$ be a dense extendable function and let $A$ be a first category set such that $I \backslash A$ is $g$-negligible. Put $h_{0}=\mathrm{id}_{I}$. By Lemma 3 , for every ordinal $\alpha<\kappa=\operatorname{card}(\Sigma)$ there exists a homeomorphism $h_{\alpha}: I \rightarrow I$ such that $h_{\alpha}(A) \cap \bigcup_{\beta<\alpha} h_{\beta}(A)=\emptyset$. By Corollary 1, all functions $g_{\alpha}=g \circ h_{\alpha}^{-1}$ are extendable. Let $\varphi: \Sigma \rightarrow \kappa$ be a bijection. By Lemma 2, the following functions are extendable:

$$
f_{\sigma}(x)=\left\{\begin{aligned}
g_{\varphi(\sigma)}(x) & \text { for } x \in h_{\varphi(\sigma)}(A), \\
f(x) & \text { for } x \notin h_{\varphi(\sigma)}(A) .
\end{aligned}\right.
$$

It is easy to verify that $f$ is the discrete limit of $\left(f_{\sigma}\right)_{\sigma \in \Sigma}$. In particular, if $\Sigma=\omega_{0}$, we obtain Theorem 3 of [13].

Corollary 2 Each function $f: I \rightarrow I$ is the pointwise limit of a sequence of extendable functions $f_{n}: I \rightarrow I$.

For $\Sigma=\omega_{1}$ we have the following corollary.

Corollary 3 Each function $f: I \rightarrow I$ is the transfinite limit of a sequence of extendable functions $f_{\alpha}: I \rightarrow I, \alpha<\omega_{1}{ }^{4}$

\footnotetext{
${ }^{3}$ The analogous result on almost continuous functions is proved in [11].

${ }^{4}$ The analogous result for almost continuous functions is proved in [5].
} 
Remark There is only one problem to obtain analogous results for any function $f: I \rightarrow \mathbb{R}$ : we are unable to construct an extendable connectivity function $g$ which is dense in $I \times \mathbb{R}$. Added in the proof.

(1) I was recently informed by Marek Balcerzak that Lemma 3 in my paper follows easily from Lemma 2 in The homeomorphic transformation of c-sets into $d$-sets by W. J. Gorman III (Proc. Amer. Math. Soc., 17 (1966), 825-830).

(2) Chris Ciesielski informed me during the latest Summer Symposium in Real Analysis, Erice, June 1995, that he with Irek Recław and, independently, Harvey Rosen had constructed an extendable function which is dense in $I \times \mathbb{R}$.

\section{References}

[1] J. B. Brown, Negligible sets for real connectivity functions, Proc. Amer. Math. Soc., 24 (1970), 263-269.

[2] J. B. Brown, Totally discontinuous connectivity functions, Colloq. Math., 23 (1971), 53-60.

[3] U. B. Darji and P. D. Humke, Every bounded function is the sum of three almost continuous bounded functions, Real Anal. Exchange, 20 (1994-95), $367-370$.

[4] R. G. Gibson and F. Roush, Connectivity functions defined on $I^{n}$, Colloq. Math., 55 (1988), 41-44.

[5] Z. Grande, Quelques remarques sur les fonctions presque continues, Probl. Mat., 10 (1988), 59-70.

[6] M. R. Hagan, Equivalence of connectivity maps and peripherally continuous transformations, Proc. Amer. Math. Soc., 17 (1966), 175-177.

[7] H. B. Hoyle, III, Connectivity maps and almost continuous functions, Duke Math. J., 37 (1970), 671-680.

[8] K. R. Kellum, Almost continuity and connectivity - sometimes it's as easy to prove a stronger result, Real Anal. Exchange, 8 (1982-83), $244-252$.

[9] K. R. Kellum and B. D. Garret, Almost continuous real functions, Proc. Amer. Math. Soc., 33 (1972), 181-184. 
[10] T. Natkaniec, On lattices generated by Darboux functions, Bull. Polish Acad. Sci. Math., 35 (1987), 549-552.

[11] T. Natkaniec, Almost Continuity, Real Anal. Exchange, 17 (1991-92), $462-520$.

[12] H. Rosen, R. G. Gibson and F. Roush, Extendable functions and almost continuous functions with a perfect road, Real Anal. Exchange, 17 (1991-92), 248-257.

[13] H. Rosen, Limits and sums of extendable connectivity functions, Real Anal. Exchange, 20 (1994-95), 183-192.

[14] W. Sierpiński, Sur les suites transfinies convergentes de fonctions de Baire, Fund. Math., 1 (1920), 132-141.

[15] J. R. Stallings, Fixed point theorems for connectivity maps, Fund. Math., 47 (1959), 249-263. 